\title{
A Rare Case of Primary Hepatic Adenosquamous Carcinoma Mimicking Liver Abscess
}

\author{
Xu Liu ${ }^{3,4}$, Yong-Qi Liu", Wei Liu ${ }^{1,2}$ \\ ${ }^{1}$ Institute of Digestive Disease, China Three Gorges University, Yichang, China \\ ${ }^{2}$ Department of Gastroenterology, Yichang Central People's Hospital, Yichang, China \\ ${ }^{3}$ Institute of Pathology, China Three Gorges University, Yichang, China \\ ${ }^{4}$ Department of Pathology, Yichang Central People's Hospital, Yichang, China
}

*Correspondence to: Wei Liu, Ph.D., Institute of Digestive Disease, China Three Gorges University, 8 Daxue Road, Yichang 443000, China; E-mail: liuwei@ctgu.edu.cn Received: Oct 19 $9^{\text {th }}, 2021$; Accepted: Oct 28 ${ }^{\text {th }}, 2021$; Published: Nov $2^{\text {nd }}, 2021$

Citation: Xu Liu, Yong-Qi Liu, Wei Liu. A rare case of primary hepatic adenosquamous carcinoma mimicking liver abscess. Gastro Open A Open J. 2021; 2(2): 5253. doi: 10.33169/gastro.GOAOJ-2-113

\section{INTRODUCTION}

A 61-year-old woman with a 3-week history of chills, fevers to $39^{\circ} \mathrm{C}$, anorexia, weight loss, and fatigue was referred to our hospital due to the progressive discomforts over the last week. Her documented medical history was notable for well-controlled type 2 diabetes. Physical examination was unremarkable. Notable laboratory tests of the blood revealed a hemoglobin level of $90 \mathrm{~g} / \mathrm{L}$, a white blood cell count of $11.7 \times 109 / \mathrm{L}$ with neutrophil $90.7 \%$, albumin $31.6 \mathrm{~g} / \mathrm{L}$, gamma glutamyl transferase $331 \mathrm{IU} / \mathrm{L}$, alkaline phosphatase 748 IU/L. Direct microbiologic detection and cultures were negative for hepatitis $\mathrm{B}$ and $\mathrm{C}$ virus, bacterial and fungal pathogens, and human immunodeficiency virus. A computed tomography scan of the chest was unremarkable. Abdominal contrast-enhanced computed tomography discovered an ill-defined and sub-circular lowdensity region measuring $7.6 \times 6.1 \mathrm{~cm}$ with patchy enhancement in the right lobe of liver (Figure A), which implied a liver abscess. Empiric antibiotics were changed to meropenem combined with metronidazole on day three of hospitalisation. However, the fever did not alleviate with poorly therapeutic effect. Ultrasound-guided biopsy was performed and purulent fluid was collected to make a definitive diagnosis of the liver mass. Surprisingly, The histopathological detection of a needle biopsy sample confirmed neoplastic necrosis with negative bacterial and fungal culture of the purulent fluid. The patient then underwent right hemihepatectomy and histopathologic examination further revealed that the lesion included a mixture of a poorly differentiated adenocarcinoma with mucin production and squamous cell carcinoma with keratinization, as well as large patchy necrosis (Figure B). On immunostaining, the lesion stained strongly with CK5/6, P63, and P40, and was also partially positive for CK7, CK8/18, and Cam5.2 with negative staining for hepatocyte and alpha-fetoprotein, which were consistent with adenosquamous carcinoma of the liver. Primary hepatic adenosquamous carcinoma is a rare variant of intrahepatic cholangiocarcinoma with approximately 10 cases reported within 5 years. ${ }^{1,2}$ The pathogenesis of adenosquamous carcinoma of the liver is largely unknown owing to limited data in the literature. Fever, jaundice, weight loss, anorexia, and abdominal pain are the most frequently reported clinical manifestations. ${ }^{3}$ Adenosquamous carcinoma of the liver usually presents atypical imaging characteristics, which is difficult to distinguish it from liver abscess, hepatocellular carcinoma, and liver metastasis. Adenosquamous carcinoma of the liver is a highly aggressive neoplasm and the median survival time is 6 months after surgery. ${ }^{4}$ Therefore, preoperative diagnosis is of vital importance to predict a poor prognosis. Despite postoperative chemoradiation, the patient passed away 7 months after surgery.

Figure 1: A Rare Case of Primary Hepatic Adenosquamous Carcinoma Mimicking Liver Abscess. A. Image findings of the tumor. B. Histopathologic findings of the adenosquamous carcinoma of the liver.

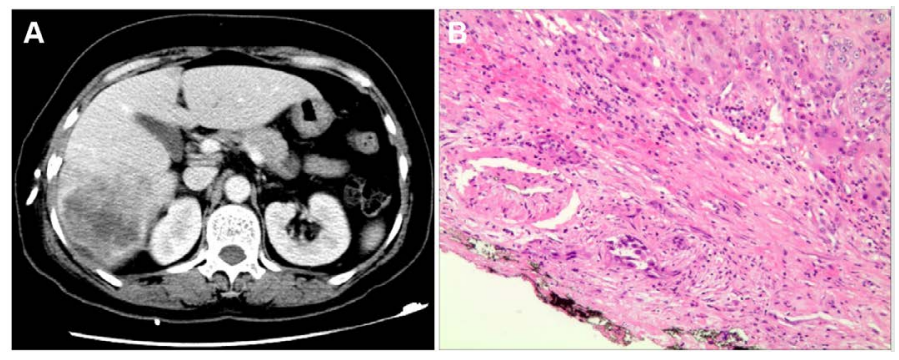

\section{FUNDING}

This work was supported by National Natural Science Foundation of China (31600134).

\section{AUTHOR'S CONTRIBUTIONS}

Collection of data: Xu Liu

Manuscript preparation and writing: Yong-Qi Liu

Final approval of the manuscript: Wei Liu 


\section{CONFLICTS OF INTEREST}

None.

\section{REFERENCES}

1. Shimizu S, Oshita A, Tashiro H, et al. Synchronous double cancers of primary hepatic adenosquamous carcinoma and hepatocellular carcinoma: report of a case. Surg Today,.2013, 43(4): 418-423.

2. Nam KH, Kim JY. Primary adenosquamous carcinoma of the liver: a case report. Clin Mol Hepatol. 2016, 22(4): 503-508.
3. Gao S, Chen D, Huang L, et al. Primary adenosquamous carcinoma of the liver: a case report and review of the literature. Int J Clin Exp Pathol. 2015, 8(8): 9687-9692.

4. Yeh CN, Jan YY, Chen MF. Adenosquamous carcinoma of the liver: clinicopathologic features in 12 patients and review of the literature. Int Surg. 2002, 87(2): 125-129. 